\title{
Critical values established in isolation without appropriate escalation policies or sophisticated algorithms can lead to tragic consequences
}

\author{
Abbreviations: CLIA, clinical laboratory improvement \\ amendments; JCAHO, joint commission on accrediting of healthcare \\ organizations; CAP, college of american pathologist; LIS, laboratory \\ information system
}

\section{Editorial}

In an article published in MLO Med Lab Obs, "When to panic over abnormal values" in 1972, George Lundberg brought the concept of critical values to the attention of the laboratory community. In many respects his definition of what constitutes a critical value then is still relevant today. He defined a critical value as a laboratory result, which suggests the patient is in imminent danger unless appropriate therapy is initiated immediately. Since Lundberg's observations in the 1970s, his concept of establishing critical values and the laboratory systems for reporting them have been established in laboratories throughout the world.

The Clinical Laboratory Improvement Amendments (CLIA) in 1988 expanded Lundberg's concept. Congress passed the CLIA in 1988 establishing quality standards for all laboratory testing to ensure the accuracy, reliability and timeliness of patient test results regardless of where the test was performed. In the United States, laboratory accrediting agencies, such as the Joint Commission on Accrediting of Healthcare Organizations (JCAHO) and the College of American Pathologist (CAP) have made critical value reporting part of the requirements for accreditation. As an example, the JCAHO states: "Measure, assess, and if appropriate, take action to improve the timeliness of reporting and the timeliness of receipt by the responsible caregiver of critical values." The CAP states: "Does the laboratory have a procedure for immediate notification of a physician (or other clinical personnel responsible for patient care) when results of certain, tests fall within established 'alert' or 'critical' ranges". Over the past decades much attention has been made regarding patient safety and how laboratory critical values and their reporting have affected the issue of patient safety.

The purpose of this editorial is not to review the formulation of a critical value list, non-critical "critical values", setting goals and measuring your laboratory performance, establishing up-to-date, clinically oriented algorithms, escalation policy and how outpatient critical values are reported, but to show what can occur when these goals are not well thought out or implemented, the consequences of which can have a negative impact on patient safety. For laboratory values to be clinical meaningful an escalation policy and or a sophisticated algorithm should be in place, which interpret laboratory values, that on face are not in themselves critical, but taken in concert with other values, and if provided, the patients underlying medical condition, will recognize these values are 'critical' for they portend a life-threatening situation for the patient. The following case will illustrate what can occur when these goals are not met.

\author{
Volume I Issue I - 2015 \\ William A Cox \\ Department of Forensic Pathology and Neuropathology, \\ Delaware State Medical Examiner's Office, USA
}

Correspondence: William A Cox, Department of Forensic Pathology and Neuropathology, Delaware State Medical Examiner's Office, P.O.X 28, Doylestown, PA I890I, USA, Tel 267-373-7850 Email william.coxmd@gmail.com

Received: May 19, 2015 | Published: May 25, 2015

The case is of a young female who went to an outpatient medical facility because of the onset of vomiting, sweating, diffuse abdominal pain accompanied by rapid shallow breathing (Kussmaul). A review of her medical history revealed she was a type II diabetic. She was a non-smoker and only drank alcohol on a rare occasion. Following a physical exam by a physician, she had blood drawn for a CBC with differential and a basic metabolic panel, which was subsequently sent to a laboratory by carrier. The results of the laboratory tests were faxed to the outpatient medical facility early that evening. Due to the fact the medical facility was closed, the results sat in the fax machine until they were retrieved at approximately 10:00am the following morning. A supine plain $\mathrm{x}$-ray of her abdomen was also taken, which was interpreted by the physician as being consistent with constipation. The patient was discharged with a diagnosis of a viral gastroenteritis and given prescriptions for Tigan suppositories, Bentyl and was placed on a BRAT diet. She was told there was nothing to worry about, however, if she did not fell better within the next two to three days she was to contact her personal physician. The patient was found the next morning by her mother deceased, approximately fifteen to sixteen hours after leaving the outpatient facility. A physician upon reviewing the laboratory results immediately called the patient to check on her wellbeing only to find the patient had died.

The laboratory values were as follows: WBC count 24THOU/ ul(4.0-11.0); Platelet 474THOU/ul(150-450); Red Blood Count 5.65mil/ul(4.00-5.40);MCV 79.2fL(80.0-100.0);Granulocyte, ABS 17.33THOU/ul(2.0-7.5); Lymphocyte, ABS 5.61THOU/ul (1.5-4.5); BUN/Creatinine Ratio 26(10-25); Glucose 388mg/dl(65-99); Carbon Dioxide $14 \mathrm{mmol} / \mathrm{L}(22-33)$; Sodium $131 \mathrm{mmol} / \mathrm{L}(136-145)$; and Chloride $94 \mathrm{mmol} / \mathrm{L}(98-107)$.

None of these values in isolation rose to the level of critical values as determined by the laboratories existing critical value list. However, looking at these values in concert, a sophisticated algorithm or a person with the appropriate medical educational background involved in the reporting process, would have recognized these values in concert raised them to the level of critical values and thus, the physician, and if necessary the patient, be immediately notified. 
The laboratory's policy for a critical value for glucose was $800 \mathrm{mg} / \mathrm{dl}$ and for an urgent value (value which was deemed not life threatening) was $500 \mathrm{mg} / \mathrm{dl}$. Looking at the above clinical (premortem) values in isolation, most especially the glucose value, none fell into the laboratory's critical value designation hence, these values were not called. It should also be noted the laboratory had no critical value for carbon dioxide. To further complicate this situation the laboratory had no escalation policy, that is, there was no one in the reporting process that could make a medical decision. Laboratory technicians do not have sufficient expertise to make medical decisions. If the laboratory chooses to place a technician only in the reporting process you are placing the technician and the laboratory in a potentially precarious position, which this case clearly demonstrated. Also, the laboratory did not make use of sophisticated algorithms, which could have assessed the values in concert and placed them in the critical value range.

An autopsy was performed later that morning. It was the opinion of the medical examiner the patient had died from hemorrhagic pancreatitis complicated by peritonitis. Vitreous fluid was drawn during the autopsy revealing the following: creatinine $1.1 \mathrm{mg} / \mathrm{dl}$ $(0.6-1.3 \mathrm{mg} / \mathrm{dl})$; sodium $152 \mathrm{mmol} / \mathrm{L}(135-150 \mathrm{mmol} / \mathrm{L})$; potassium $8.2 \mathrm{mmol} / \mathrm{L}(<15 \mathrm{mmol} / \mathrm{L}$ is considered normal); chloride $107 \mathrm{mmol} / \mathrm{L}$ $(105-135 \mathrm{mmol} / \mathrm{L})$; glucose $479 \mathrm{mg} / \mathrm{dl} \quad(<200 \mathrm{mg} / \mathrm{dl}$ is considered normal; postmortem vitreous glucose concentrations $>200 \mathrm{mg} / \mathrm{dl}$ are associated with hyperglycemia); and urea nitrogen $57 \mathrm{mg} / \mathrm{dl}(8-20 \mathrm{mg} /$ dl). Although, the patient was a type II diabetic and had died from hemorrhagic pancreatitis complicated by peritonitis, the Medical Examiner was apparently unaware type II diabetics have a 2.8 times chance of developing pancreatitis. He also did not appreciate although diabetic ketoacidosis (DKA) is generally seen in type I patients, DKA is seen with increasing frequency in obese type II patients. The patient was considered obese due to the fact she was $5^{\prime} 7^{\prime}$ " in height and weighed 219 pounds consequently, he did not order vitreous ketones.

A sophisticated algorithm or a person with sufficient medical expertise involved in the reporting process would have recognized, although a glucose of $388 \mathrm{mg} / \mathrm{dl}$ was not a critical value according to the laboratories critical value list, taken in concert with a carbon dioxide of $14 \mathrm{mmol} / \mathrm{L}$ was a reflection of a decrease in bicarbonate due to its buffering action against the presence of acids, and thus would have appreciated the patient had a metabolic acidosis. Further, using a nomogram, plotting carbon dioxide against $\mathrm{pH}$ would have revealed a $\mathrm{pH}$ of 7.125, which would have placed the patient in the moderate severe category of metabolic acidosis, which would have suggested a bicarbonate in the range of $10-15 \mathrm{mmol} / \mathrm{L}$ (normal $22-28 \mathrm{mmol} / \mathrm{L}$ ). Doing a calculation for anion gap would have shown the patient had a high anion gap and thus further supported a diagnosis of metabolic acidosis. Thus, the patients glucose of $388 \mathrm{mg} / \mathrm{dl}$ and carbon dioxide of $14 \mathrm{mmol} / \mathrm{L}$ were critical values occurring in a patient with type II diabetes suggesting the presence of DKA. This conclusion would have been supported by the fact a glucose of 388 falls within the range of glucose levels seen in DKA, which may range from 250 to more than $1000 \mathrm{mg} / \mathrm{dl}$. In addition, the presence of a carbon dioxide of $14 \mathrm{mmol} / \mathrm{L}$ was a reflection of bicarbonate being decreased through its buffering action against the presence of acids, in this case, ketoacids. It would have also been recognized the low carbon dioxide of $14 \mathrm{mmol} / \mathrm{L}$ was an attempt at compensatory respiratory alkalosis, which was the result of the patient's Kussmaul respirations. They would have also realized a leukocytosis of 24,000 was also consistent with DKA. At the time these values were reported the autopsy had not been done consequently, they were unaware of the diagnosis of acute hemorrhagic pancreatitis complicated by peritonitis, which also would have caused an elevated leukocyte count. In addition they would have noted the low sodium concentration represented an osmotic flux of water from the intracellular space to the extracellular space in the presence of hyperglycemia. They further would have anticipated losses of sodium, magnesium, and phosphorus to occur as the amount of total body water decreased due to the development of hypovolemia. It would have been recognized serum levels of these ions could become normal or elevated owing to hypovolemia as was seen in the postmortem vitreous, which showed a sodium of $152 \mathrm{mmol} / \mathrm{L}$. Hypovolemia and dehydration would also account for increased values of hematocrit.

What must be remembered is a patient with DKA has a fluid deficit of approximately 6Liters in total body fluids, which is why initial fluid therapy is directed toward expansions of the intravascular and extra vascular volume and thus restoration of renal perfusion. In addition the patient had acute hemorrhagic pancreatitis complicated by peritonitis, which resulted in an additional loss of $800 \mathrm{ml}$ of fluid into the peritoneal cavity. To further support the existence of dehydration the patient had a premortem BUN-to-creatinine ratio of 26 , which is a reflection of BUN re absorption being increased and thus, it is disproportionately elevated relative to creatinine in serum thus, dehydration is suspected.

A sophisticated algorithm or an appropriate escalation policy would have noted the patient's potassium level of $3.8 \mathrm{mmol} / \mathrm{L}$ (3.45.3), although in the normal range, was low normal. Such a potassium value in the context of this patient's laboratory values would have raised a red flag for it suggested a severe deficit in total body potassium. Physiologically potassium is constantly leaking into the vascular space through diffusion and is transported intracellularly via the sodium-potassium pump. However, this active transport mechanism requires insulin, and in the presence of ketoacidosis, the absence of insulin results in a transient hyperkalemia for potassium cannot be actively transported back into the cell. The excess potassium is excreted into the urine, eventually leading to a net potassium loss. Such a net loss of potassium requires vigorous potassium replacement, however, under careful cardiac monitoring, especially considering the treatment of DKA, which includes giving insulin the result of which will drive potassium ion from the extracellularly compartment into the intracellular compartment, which in turn will further lower potassium and can lead to a fatal cardiac dysrhythmia. Consequently, the patients low normal potassium level in actuality represented a critical value.

The ability to interpret laboratory reports, whether accomplished through an appropriate escalation policy, using someone in the reporting process who can make medical decisions and or a sophisticated algorithm, are an essential component of laboratory practice, which can transform laboratory data into clinically useful information. The Laboratory Information System (LIS) can be used to transmit pathologist generated comments; may automatically produce interpretations on reports based on a predefined algorithms; or may serve as a complex expert system, suggesting interpretive comments to the clinician. Some sophisticated algorithms will allow one to customize clinical alerts by physicians or specialty to more accurately identify patients who may have results that are potentially serious given their condition, for it is well recognized a single set of clinical alerts does not take into account a patent's underlying condition, which was the unfortunate situation in this patient. Had such a sophisticated algorithm been part of this LIS or had this laboratory had an escalation 
policy in which there was a person involved in the reporting process who could make medical decisions, and even potentially call the patient, there is every reason to believe this young female patient would never had died from DKA due to acute pancreatitis. With the ready availability of quality medical care in this country virtually no one should die from acute pancreatitis or DKA.

When assessing a laboratories critical value policies we must ask ourselves, do these policies improve outcome, are they medically sound, and above all do they meet the standard of care. What is more important, is the laboratory cognizant of the ever evolving sophisticated algorithms, which can further improve patient safety. It must always be remembered, critical values are critical for they have the potential to threaten the patients very existence thus, it is most important laboratories have policies and procedures, which provide for patients wellbeing and safety.

\section{Acknowledgements}

None.

\section{Conflict of interest}

The author declares no conflict of interest. 